\title{
Generalized Second-Order Mixed Symmetric Duality in Nondifferentiable Mathematical Programming
}

\author{
Ravi P. Agarwal, ${ }^{1}$ Izhar Ahmad, $^{1}$ S. K. Gupta, ${ }^{2}$ and N. Kailey ${ }^{3}$ \\ ${ }^{1}$ Department of Mathematics and Statistics, King Fahd University of Petroleum and Minerals, \\ Dhahran 31261, Saudi Arabia \\ ${ }^{2}$ Department of Mathematics, Indian Institute of Technology Patna, Patna 800 013, India \\ ${ }^{3}$ School of Mathematics and Computer Applications, Thapar University, Patiala 147 004, India
}

Correspondence should be addressed to Izhar Ahmad, izharmaths@hotmail.com

Received 30 December 2010; Accepted 24 January 2011

Academic Editor: Elena Litsyn

Copyright (C) 2011 Ravi P. Agarwal et al. This is an open access article distributed under the Creative Commons Attribution License, which permits unrestricted use, distribution, and reproduction in any medium, provided the original work is properly cited.

This paper is concerned with a pair of second-order mixed symmetric dual programs involving nondifferentiable functions. Weak, strong, and converse duality theorems are proved for aforementioned pair using the notion of second-order $F$-convexity/pseudoconvexity assumptions.

\section{Introduction}

Duality is a fruitful theory in mathematical programming and is useful both theoretically and practically. Duality as used in our daily life means the sort of harmony of two opposite or complementary parts through which they integrate into a whole. Symmetry is bound up with duality and, in particular, is significant in mathematics. The problem of optimizing a numerical function of one or more variables subject to constraints on the variables is called the mathematical programming, or constrained optimization, problem. When either the objective function or one or more of the constraints are nonlinear, the programming problem is called a nonlinear programming problem, a discipline playing an increasingly imperative role in such diverse fields as operations research and management science, engineering, economics, system analysis, and computer science.

Dantzig et al. [1], Mond [2], and Bazaraa and Goode [3] studied symmetric duality in nonlinear programming. Later, Chandra and Husain [4] formulated a pair of Wolfetype nondifferentiable symmetric dual programs and proved duality results under 
convexity/concavity assumptions. Subsequently, Chandra et al. [5] weakened these assumptions to pseudoconvexity/pseudoconcavity. Mond and Schechter [6] presented two symmetric dual pairs involving nondifferentiable functions. Kumar and Bhatia [7] discussed multiobjective symmetric duality by using a nonlinear vector-valued function of two variables corresponding to various objectives.

Mangasarian [8] presented a dual problem associated with a primal nonlinear programming problem that involves second derivatives of the function constituting the primal problem. The study of second-order duality is significant, as it can provide a lower bound to the infimum of a primal optimization problem when it is difficult to find a feasible solution for the first-order dual. Bector and Chandra [9] achieved duality results for a pair of Mond-Weir-type second-order symmetric dual nonlinear programs. Hou and Yang [10] formulated a pair of second-order symmetric dual nondifferentiable programs and established duality theorems under second-order $F$-pseudoconvexity assumptions.

Chandra et al. [11] and Yang et al. [12] discussed a mixed symmetric dual formulation for a nonlinear programming problem and for a class of nondifferentiable nonlinear programming problems, respectively. Later on, Ahmad [13] formulated mixed type symmetric dual in multiobjective programming problems ignoring nonnegativity restrictions of Bector et al. [14].

In this paper, a pair of second-order mixed symmetric dual programs is presented for a class of nondifferentiable nonlinear programming problems. Weak, strong, and converse duality theorems are proved using the notion of second-order $F$-convexity/pseudoconvexity assumptions. These results generalize the known work in [6, 10-12, 15-17].

\section{Preliminaries}

In this section, we presented some of the basic definitions used in the paper.

Definition 2.1. Let $C$ be a compact convex set in $R^{n}$. The support function of $C$ is defined by

$$
S(x \mid C)=\max \left\{x^{T} y: y \in C\right\}
$$

A support function, being convex and everywhere finite, has a subdifferential, that is, there exists $z \in R^{n}$ such that

$$
S(y \mid C) \geq S(x \mid C)+z^{T}(y-x), \quad \forall y \in C
$$

The subdifferential of $S(x \mid C)$ is given by

$$
\partial S(x \mid C)=\left\{z \in C: z^{T} x=S(x \mid C)\right\} .
$$

For any set $S \subset R^{n}$, the normal cone to $S$ at a point $x \in S$ is defined by

$$
N_{S}(x)=\left\{y \in R^{n}: y^{T}(z-x) \leq 0, \forall z \in S\right\}
$$


It can be easily seen that for a compact convex set $C, y$ is in $N_{C}(x)$ if and only if $S(y \mid C)=$ $x^{T} y$, or equivalently, $x$ is in $\partial S(y \mid C)$.

Definition 2.2. A functional $F: X \times X \times R^{n} \mapsto R$ (where $X \subseteq R^{n}$ ) is sublinear with respect to the third variable if for all $(x, u) \in X \times X$,

(i) $F\left(x, u ; a_{1}+a_{2}\right) \leq F\left(x, u ; a_{1}\right)+F\left(x, u ; a_{2}\right)$ for all $a_{1}, a_{2} \in R^{n}$,

(ii) $F(x, u ; \alpha a)=\alpha F(x, u ; a)$, for all $\alpha \in R_{+}$and for all $a \in R^{n}$.

Let $\psi: X \mapsto R$ be a real-valued twice differentiable function.

Definition 2.3. $\psi$ is said to be second-order $F$-convex at $u \in X$ with respect to $q \in R^{n}$, if for all $x \in X$,

$$
\psi(x)-\psi(u)+\frac{1}{2} q^{T} \nabla_{x x} \psi(u) q \geq F\left(x, u ; \nabla_{x} \psi(u)+\nabla_{x x} \psi(u) q\right)
$$

Definition 2.4. $\psi$ is said to be second-order F-pseudoconvex at $u \in X$ with respect to $q \in R^{n}$, if for all $x \in X$,

$$
F\left(x, u ; \nabla_{x} \psi(u)+\nabla_{x x} \psi(u) q\right) \geq 0 \Longrightarrow \psi(x) \geq \psi(u)-\frac{1}{2} q^{T} \nabla_{x x} \psi(u) q
$$

$\psi$ is second-order $F$-concave/pseudoconcave at $u \in X$ with respect to $q \in R^{n}$ if $-\psi$ is secondorder $F$-convex/pseudoconvex at $u \in X$ with respect to $q \in R^{n}$.

\section{Second-Order Mixed Nondifferentiable Symmetric Dual Programs}

For $N=\{1,2, \ldots, n\}$ and $M=\{1,2, \ldots, m\}$, let $J_{1} \subseteq N, K_{1} \subseteq M, J_{2}=N \backslash J_{1}$, and $K_{2}=M \backslash K_{1}$. Let $\left|J_{1}\right|$ denote the number of elements in $J_{1}$. The other symbols $\left|J_{2}\right|,\left|K_{1}\right|$ and $\left|K_{2}\right|$ are defined similarly. Let $x^{1} \in R^{\left|J_{1}\right|}, x^{2} \in R^{\left|J_{2}\right|}$. Then, any $x \in R^{n}$ can be written as $\left(x^{1}, x^{2}\right)$. Similarly, for $y^{1} \in R^{\left|K_{1}\right|}, y^{2} \in R^{\left|K_{2}\right|}, y \in R^{m}$ can be written as $\left(y^{1}, y^{2}\right)$. It may be noted here that if $J_{1}=\emptyset$, then $\left|J_{1}\right|=0, J_{2}=N$, and therefore $\left|J_{2}\right|=n$. In this case, $R^{\left|J_{1}\right|}, R^{\left|J_{2}\right|}$ and $R^{\left|J_{1}\right|} \times R^{\left|K_{1}\right|}$ will be zerodimensional, $n$-dimensional and $\left|K_{1}\right|$-dimensional Euclidean spaces, respectively. The other situations are $J_{2}=\emptyset, K_{1}=\emptyset$ or $K_{2}=\emptyset$.

Now we formulate the following pair of mixed nondifferentiable second-order symmetric dual programs and discuss their duality results. 
Primal problem (SMNP)

minimize

$$
\begin{aligned}
& G\left(x^{1}, y^{1}, x^{2}, y^{2}, z^{2}, p, r\right) \\
&= f\left(x^{1}, y^{1}\right)+S\left(x^{1} \mid C_{1}\right)+g\left(x^{2}, y^{2}\right)+S\left(x^{2} \mid C_{2}\right)-\left(y^{2}\right)^{T} z^{2} \\
&-\left(y^{1}\right)^{T}\left[\nabla_{y^{1}} f\left(x^{1}, y^{1}\right)+\nabla_{y^{1} y^{1}} f\left(x^{1}, y^{1}\right) p\right]-\frac{1}{2} p^{T} \nabla_{y^{1} y^{1}} f\left(x^{1}, y^{1}\right) p \\
&-\frac{1}{2} r^{T} \nabla_{y^{2} y^{2}} g\left(x^{2}, y^{2}\right) r
\end{aligned}
$$

subject to

$$
\begin{gathered}
\nabla_{y^{1}} f\left(x^{1}, y^{1}\right)-z^{1}+\nabla_{y^{1} y^{1}} f\left(x^{1}, y^{1}\right) p \leq 0, \\
\nabla_{y^{2}} g\left(x^{2}, y^{2}\right)-z^{2}+\nabla_{y^{2} y^{2}} g\left(x^{2}, y^{2}\right) r \leq 0 \\
\left(y^{2}\right)^{T}\left[\nabla_{y^{2}} g\left(x^{2}, y^{2}\right)-z^{2}+\nabla_{y^{2} y^{2}} g\left(x^{2}, y^{2}\right) r\right] \geq 0, \\
z^{1} \in D_{1}, \quad z^{2} \in D_{2} .
\end{gathered}
$$

Dual problem (SMND)

maximize

$$
\begin{aligned}
& H\left(u^{1}, v^{1}, u^{2}, v^{2}, w^{2}, q, s\right) \\
&= f\left(u^{1}, v^{1}\right)-S\left(v^{1} \mid D_{1}\right)+g\left(u^{2}, v^{2}\right)-S\left(v^{2} \mid D_{2}\right)+\left(u^{2}\right)^{T} w^{2} \\
&-\left(u^{1}\right)^{T}\left[\nabla_{x^{1}} f\left(u^{1}, v^{1}\right)+\nabla_{x^{1} x^{1}} f\left(u^{1}, v^{1}\right) q\right]-\frac{1}{2} q^{T} \nabla_{x^{1} x^{1}} f\left(u^{1}, v^{1}\right) q \\
&-\frac{1}{2} s^{T} \nabla_{x^{2} x^{2}} g\left(u^{2}, v^{2}\right) s,
\end{aligned}
$$

subject to

$$
\begin{gathered}
\nabla_{x^{1}} f\left(u^{1}, v^{1}\right)+w^{1}+\nabla_{x^{1} x^{1}} f\left(u^{1}, v^{1}\right) q \geq 0, \\
\nabla_{x^{2}} g\left(u^{2}, v^{2}\right)+w^{2}+\nabla_{x^{2} x^{2}} g\left(u^{2}, v^{2}\right) s \geq 0, \\
\left(u^{2}\right)^{T}\left[\nabla_{x^{2}} g\left(u^{2}, v^{2}\right)+w^{2}+\nabla_{x^{2} x^{2}} g\left(u^{2}, v^{2}\right) s\right] \leq 0, \\
w^{1} \in C_{1}, \quad w^{2} \in C_{2},
\end{gathered}
$$


where

(i) $f: R^{\left|J_{1}\right|} \times R^{\left|K_{1}\right|} \rightarrow R$ and $g: R^{\left|J_{2}\right|} \times R^{\left|K_{2}\right|} \rightarrow R$ are differentiable functions,

(ii) $C_{1}, C_{2}, D_{1}$ and $D_{2}$ are compact convex sets in $R^{\left|J_{1}\right|}, R^{\left|J_{2}\right|}, R^{\left|K_{1}\right|}$ and $R^{\left|K_{2}\right|}$, respectively,

(iii) $p \in R^{\left|K_{1}\right|}, r \in R^{\left|K_{2}\right|}, q \in R^{\left|J_{1}\right|}$ and $s \in R^{\left|J_{2}\right|}$.

Theorem 3.1 (Weak duality). Let $\left(x^{1}, y^{1}, x^{2}, y^{2}, z^{1}, z^{2}, p, r\right)$ be feasible for (SMNP) and $\left(u^{1}, v^{1}, u^{2}, v^{2}, w^{1}, w^{2}, q, s\right)$ be feasible for (SMND). Let the sublinear functionals $F_{1}: R^{\left|J_{1}\right|} \times R^{\left|J_{1}\right|} \times$ $R^{\left|J_{1}\right|} \mapsto R, F_{2}: R^{\left|K_{1}\right|} \times R^{\left|K_{1}\right|} \times R^{\left|K_{1}\right|} \mapsto R, G_{1}: R^{\left|J_{2}\right|} \times R^{\left|J_{2}\right|} \times R^{\left|J_{2}\right|} \mapsto R$ and $G_{2}: R^{\left|K_{2}\right|} \times R^{\left|K_{2}\right|} \times R^{\left|K_{2}\right|} \mapsto R$ satisfy the following conditions:

$$
\begin{array}{ll}
F_{1}\left(x^{1}, u^{1} ; a^{1}\right)+\left(a^{1}\right)^{T} u^{1} \geq 0, & \forall a^{1} \in R_{+}^{\left|J_{1}\right|}, \\
F_{2}\left(v^{1}, y^{1} ; a^{2}\right)+\left(a^{2}\right)^{T} y^{1} \geq 0, & \forall a^{2} \in R_{+}^{\left|K_{1}\right|}, \\
G_{1}\left(x^{2}, u^{2} ; b^{1}\right)+\left(b^{1}\right)^{T} u^{2} \geq 0, & \forall b^{1} \in R_{+}^{\left|J_{2}\right|}, \\
G_{2}\left(v^{2}, y^{2} ; b^{2}\right)+\left(b^{2}\right)^{T} y^{2} \geq 0, \quad \forall b^{2} \in R_{+}^{\left|K_{2}\right|}
\end{array}
$$

Suppose that

(i) $f\left(\cdot, v^{1}\right)+(\cdot)^{T} w^{1}$ is second-order $F_{1}$-convex at $u^{1}$, and $f\left(x^{1}, \cdot\right)-(\cdot)^{T} z^{1}$ is second-order $F_{2}$-concave at $y^{1}$,

(ii) $g\left(\cdot, v^{2}\right)+(\cdot)^{T} w^{2}$ is second-order $G_{1}-p s e u d o c o n v e x$ at $u^{2}$, and $g\left(x^{2}, \cdot\right)-(\cdot)^{T} z^{2}$ is secondorder $G_{2}$-pseudoconcave at $y^{2}$.

Then,

$$
G\left(x^{1}, y^{1}, x^{2}, y^{2}, z^{2}, p, r\right) \geq H\left(u^{1}, v^{1}, u^{2}, v^{2}, w^{2}, q, s\right) .
$$

Proof. By the second-order $F_{1}$-convexity of $f\left(\cdot, v^{1}\right)+(\cdot)^{T} w^{1}$ at $u^{1}$ and the second-order $F_{2}-$ concavity of $f\left(x^{1}, \cdot\right)-(\cdot)^{T} z^{1}$ at $y^{1}$, we have

$$
\begin{gathered}
f\left(x^{1}, v^{1}\right)+\left(x^{1}\right)^{T} w^{1}-f\left(u^{1}, v^{1}\right)-\left(u^{1}\right)^{T} w^{1}+\frac{1}{2} q^{T} \nabla_{x^{1} x^{1}} f\left(u^{1}, v^{1}\right) q \\
\geq F_{1}\left(x^{1}, u^{1} ; \nabla_{x^{1}} f\left(u^{1}, v^{1}\right)+w^{1}+\nabla_{x^{1} x^{1}} f\left(u^{1}, v^{1}\right) q\right), \\
f\left(x^{1}, y^{1}\right)-\left(y^{1}\right)^{T} z_{1}-f\left(x^{1}, v^{1}\right)+\left(v^{1}\right)^{T} z_{1}-\frac{1}{2} p^{T} \nabla_{y^{1} y^{1}} f\left(x^{1}, y^{1}\right) p \\
\geq F_{2}\left(v^{1}, y^{1} ;-\left(\nabla_{y^{1}} f\left(x^{1}, y^{1}\right)-z^{1}+\nabla_{y^{1} y^{1}} f\left(x^{1}, y^{1}\right) p\right)\right) .
\end{gathered}
$$

Since $\left(x^{1}, y^{1}, x^{2}, y^{2}, z^{1}, z^{2}, p, r\right)$ is feasible for primal problem (SMNP) and $\left(u^{1}, v^{1}\right.$, $\left.u^{2}, v^{2}, w^{1}, w^{2}, q, s\right)$ is feasible for dual problem (SMND), by the dual constraint (3.7), 
the vector $a^{1}=\nabla_{x^{1}} f\left(u^{1}, v^{1}\right)+w^{1}+\nabla_{x^{1} x^{1}} f\left(u^{1}, v^{1}\right) q \in R_{+}^{\left|J_{1}\right|}$, and so from the hypothesis (A), we obtain

$$
F_{1}\left(x^{1}, u^{1} ; a^{1}\right)+\left(a^{1}\right)^{T} u^{1} \geq 0 .
$$

Similarly,

$$
F_{2}\left(v^{1}, y^{1} ; a^{2}\right)+\left(a^{2}\right)^{T} y^{1} \geq 0
$$

for the vector $a^{2}=-\left[\nabla_{y^{1}} f\left(x^{1}, y^{1}\right)-z^{1}+\nabla_{y^{1} y^{1}} f\left(x^{1}, y^{1}\right) p\right] \in R_{+}^{\left|K_{1}\right|}$.

Using (3.14) in (3.12) and (3.15) in (3.13), we have

$$
\begin{aligned}
& f\left(x^{1}, v^{1}\right)+\left(x^{1}\right)^{T} w^{1}-f\left(u^{1}, v^{1}\right)-\left(u^{1}\right)^{T} w^{1}+\frac{1}{2} q^{T} \nabla_{x^{1} x^{1}} f\left(u^{1}, v^{1}\right) q \geq-\left(u^{1}\right)^{T} a^{1}, \\
& f\left(x^{1}, y^{1}\right)-\left(y^{1}\right)^{T} z_{1}-f\left(x^{1}, v^{1}\right)+\left(v^{1}\right)^{T} z_{1}-\frac{1}{2} p^{T} \nabla_{y^{1} y^{1}} f\left(x^{1}, y^{1}\right) p \geq-\left(y^{1}\right)^{T} a^{2} .
\end{aligned}
$$

Adding the above two inequalities, we obtain

$$
\begin{aligned}
& f\left(x^{1}, y^{1}\right)+\left(x^{1}\right)^{T} w^{1}-\left(y^{1}\right)^{T} z_{1}+\left(y^{1}\right)^{T} a^{2}-\frac{1}{2} p^{T} \nabla_{y^{1} y^{1}} f\left(x^{1}, y^{1}\right) p \\
& \geq f\left(u^{1}, v^{1}\right)+\left(u^{1}\right)^{T} w^{1}-\left(v^{1}\right)^{T} z_{1}-\left(u^{1}\right)^{T} a^{1}-\frac{1}{2} q^{T} \nabla_{x^{1} x^{1}} f\left(u^{1}, v^{1}\right) q .
\end{aligned}
$$

Substituting the values of $a^{1}$ and $a^{2}$ in (3.17), we get

$$
\begin{aligned}
f\left(x^{1}, y^{1}\right)+\left(x^{1}\right)^{T} w^{1}-\left(y^{1}\right)^{T}\left[\nabla_{y^{1}} f\left(x^{1}, y^{1}\right)+\nabla_{y^{1} y^{1}} f\left(x^{1}, y^{1}\right) p\right]-\frac{1}{2} p^{T} \nabla_{y^{1} y^{1}} f\left(x^{1}, y^{1}\right) p \\
\geq \\
\quad f\left(u^{1}, v^{1}\right)-\left(v^{1}\right)^{T} z_{1}-\left(u^{1}\right)^{T}\left[\nabla_{x^{1}} f\left(u^{1}, v^{1}\right)+\nabla_{x^{1} x^{1}} f\left(u^{1}, v^{1}\right) q\right] \\
\quad-\frac{1}{2} q^{T} \nabla_{x^{1} x^{1}} f\left(u^{1}, v^{1}\right) q .
\end{aligned}
$$

Using $\left(x^{1}\right)^{T} w^{1} \leq S\left(x^{1} \mid C_{1}\right)$ and $\left(v^{1}\right)^{T} z^{1} \leq S\left(v^{1} \mid D_{1}\right)$, we have

$$
\begin{aligned}
f\left(x^{1}, y^{1}\right)+S\left(x^{1} \mid C_{1}\right)-\left(y^{1}\right)^{T}\left[\nabla_{y^{1}} f\left(x^{1}, y^{1}\right)+\nabla_{y^{1} y^{1}} f\left(x^{1}, y^{1}\right) p\right]-\frac{1}{2} p^{T} \nabla_{y^{1} y^{1}} f\left(x^{1}, y^{1}\right) p \\
\geq \\
\quad f\left(u^{1}, v^{1}\right)-S\left(v^{1} \mid D_{1}\right)-\left(u^{1}\right)^{T}\left[\nabla_{x^{1}} f\left(u^{1}, v^{1}\right)+\nabla_{x^{1} x^{1}} f\left(u^{1}, v^{1}\right) q\right] \\
\quad-\frac{1}{2} q^{T} \nabla_{x^{1} x^{1}} f\left(u^{1}, v^{1}\right) q .
\end{aligned}
$$


By hypothesis (C) and the dual constraint (3.8), we obtain

$$
\begin{aligned}
& G_{1}\left(x^{2}, u^{2} ; \nabla_{x^{2}} g\left(u^{2}, v^{2}\right)+w^{2}+\nabla_{x^{2} x^{2}} g\left(u^{2}, v^{2}\right) s\right) \\
& \quad \geq-\left(u^{2}\right)^{T}\left[\nabla_{x^{2}} g\left(u^{2}, v^{2}\right)+w^{2}+\nabla_{x^{2} x^{2}} g\left(u^{2}, v^{2}\right) s\right],
\end{aligned}
$$

which on using the dual constraint (3.9) yields

$$
G_{1}\left(x^{2}, u^{2} ; \nabla_{x^{2}} g\left(u^{2}, v^{2}\right)+w^{2}+\nabla_{x^{2} x^{2}} g\left(u^{2}, v^{2}\right) s\right) \geq 0
$$

Since $g\left(\cdot, v^{2}\right)+(\cdot)^{T} w^{2}$ is second-order $G_{1}$-pseudoconvex at $u^{2}$, we have

$$
g\left(x^{2}, v^{2}\right)+\left(x^{2}\right)^{T} w^{2} \geq g\left(u^{2}, v^{2}\right)+\left(u^{2}\right)^{T} w^{2}-\frac{1}{2} s^{T} \nabla_{x^{2} x^{2}} g\left(u^{2}, v^{2}\right) s .
$$

Similarly, from (3.3) and (3.4) and hypothesis (D) along with second-order $G_{2}$-pseudoconcavity of $g\left(x^{2}, \cdot\right)-(\cdot)^{T} z^{2}$ at $y^{2}$, we get

$$
g\left(x^{2}, y^{2}\right)-\left(y^{2}\right)^{T} z^{2} \geq g\left(x^{2}, v^{2}\right)-\left(v^{2}\right)^{T} z^{2}+\frac{1}{2} r^{T} \nabla_{y^{2} y^{2}} g\left(x^{2}, y^{2}\right) r
$$

Adding (3.22) and (3.23), we obtain

$$
\begin{aligned}
& g\left(x^{2}, y^{2}\right)+\left(x^{2}\right)^{T} w^{2}-\left(y^{2}\right)^{T} z^{2}-\frac{1}{2} r^{T} \nabla_{y^{2} y^{2}} g\left(x^{2}, y^{2}\right) r \\
& \geq g\left(u^{2}, v^{2}\right)+\left(u^{2}\right)^{T} w^{2}-\left(v^{2}\right)^{T} z^{2}-\frac{1}{2} s^{T} \nabla_{x^{2} x^{2}} g\left(u^{2}, v^{2}\right) s .
\end{aligned}
$$

Using $\left(x^{2}\right)^{T} w^{2} \leq S\left(x^{2} \mid C_{2}\right)$ and $\left(v^{2}\right)^{T} z^{2} \leq S\left(v^{2} \mid D_{2}\right)$, we have

$$
\begin{aligned}
& g\left(x^{2}, y^{2}\right)+S\left(x^{2} \mid C_{2}\right)-\left(y^{2}\right)^{T} z^{2}-\frac{1}{2} r^{T} \nabla_{y^{2} y^{2}} g\left(x^{2}, y^{2}\right) r \\
& \quad \geq g\left(u^{2}, v^{2}\right)+\left(u^{2}\right)^{T} w^{2}-S\left(v^{2} \mid D_{2}\right)-\frac{1}{2} s^{T} \nabla_{x^{2} x^{2}} g\left(u^{2}, v^{2}\right) s .
\end{aligned}
$$


Inequalities (3.19) and (3.25) together yield

$$
\begin{aligned}
f\left(x^{1}, y^{1}\right)+S\left(x^{1} \mid C_{1}\right)+g\left(x^{2}, y^{2}\right)+S\left(x^{2} \mid C_{2}\right)-\left(y^{2}\right)^{T} z^{2} \\
\quad-\left(y^{1}\right)^{T}\left[\nabla_{y^{1}} f\left(x^{1}, y^{1}\right)+\nabla_{y^{1} y^{1}} f\left(x^{1}, y^{1}\right) p\right]-\frac{1}{2} p^{T} \nabla_{y^{1} y^{1}} f\left(x^{1}, y^{1}\right) p-\frac{1}{2} r^{T} \nabla_{y^{2} y^{2}} g\left(x^{2}, y^{2}\right) r \\
\geq \\
\quad f\left(u^{1}, v^{1}\right)-S\left(v^{1} \mid D_{1}\right)+g\left(u^{2}, v^{2}\right)-S\left(v^{2} \mid D_{2}\right)+\left(u^{2}\right)^{T} w^{2} \\
\quad-\left(u^{1}\right)^{T}\left[\nabla_{x^{1}} f\left(u^{1}, v^{1}\right)+\nabla_{x^{1} x^{1}} f\left(u^{1}, v^{1}\right) q\right]-\frac{1}{2} q^{T} \nabla_{x^{1} x^{1}} f\left(u^{1}, v^{1}\right) q \\
\quad-\frac{1}{2} s^{T} \nabla_{x^{2} x^{2}} g\left(u^{2}, v^{2}\right) s
\end{aligned}
$$

that is, $G\left(x^{1}, y^{1}, x^{2}, y^{2}, z^{2}, p, r\right) \geq H\left(u^{1}, v^{1}, u^{2}, v^{2}, w^{2}, q, s\right)$.

Theorem 3.2 (Strong duality). Let $f: R^{\left|J_{1}\right|} \times R^{\left|K_{1}\right|} \rightarrow R$ and $g: R^{\left|J_{2}\right|} \times R^{\left|K_{2}\right|} \rightarrow R$ be differentiable functions, and let $\left(\bar{x}^{1}, \bar{y}^{1}, \bar{x}^{2}, \bar{y}^{2}, \bar{z}^{1}, \bar{z}^{2}, \bar{p}, \bar{r}\right)$ be a local optimal solution of (SMNP). Suppose that

(i) the matrix $\nabla_{y^{1} y^{1}} f\left(\bar{x}^{1}, \bar{y}^{1}\right)$ is non singular,

(ii) $\nabla_{y^{2} y^{2}} g\left(\bar{x}^{2}, \bar{y}^{2}\right)$ is positive definite, $\bar{r}^{T}\left(\nabla_{y^{2}} g\left(\bar{x}^{2}, \bar{y}^{2}\right)-\bar{z}^{2}\right) \geq 0$ or $\nabla_{y^{2} y^{2}} g\left(\bar{x}^{2}, \bar{y}^{2}\right)$ is negative definite, and $\bar{r}^{T}\left(\nabla_{y^{2}} g\left(\bar{x}^{2}, \bar{y}^{2}\right)-\bar{z}^{2}\right) \leq 0$,

(iii) $\nabla_{y^{2}} g\left(\bar{x}^{2}, \bar{y}^{2}\right)-\bar{z}^{2}+\nabla_{y^{2} y^{2}} g\left(\bar{x}^{2}, \bar{y}^{2}\right) \bar{r} \neq 0$,

(iv) one of the matrices $\left(\partial / \partial y_{i}^{1}\right)\left(\nabla_{y^{1} y^{1}} f\left(\bar{x}^{1}, \bar{y}^{1}\right)\right), i=1,2, \ldots,\left|K_{1}\right|$, is positive or negative definite.

Then, there exist $\bar{w}^{1} \in C_{1}$ and $\bar{w}^{2} \in C_{2}$ such that $\left(\bar{x}^{1}, \bar{y}^{1}, \bar{x}^{2}, \bar{y}^{2}, \bar{w}^{1}, \bar{w}^{2}, \bar{q}=0, \bar{s}=0\right)$ is feasible for (SMND), and the objective function values of (SMNP) and (SMND) are equal. Furthermore, if the assumptions of weak duality (Theorem 3.1) are satisfied for all feasible solutions of (SMNP) and (SMND), then $\left(\bar{x}^{1}, \bar{y}^{1}, \bar{x}^{2}, \bar{y}^{2}, \bar{z}^{1}, \bar{z}^{2}, \bar{p}, \bar{r}\right)$ and $\left(\bar{x}^{1}, \bar{y}^{1}, \bar{x}^{2}, \bar{y}^{2}, \bar{w}^{1}, \bar{w}^{2}, \bar{q}, \bar{s}\right)$ are global optimal solutions for (SMNP) and (SMND), respectively.

Proof. Since $\left(\bar{x}^{1}, \bar{y}^{1}, \bar{x}^{2}, \bar{y}^{2}, \bar{z}^{1}, \bar{z}^{2}, \bar{p}, \bar{r}\right)$ is a local optimal solution of (SMNP), there exist $\alpha \in R$, $\beta \in R^{\left|K_{1}\right|}, \gamma \in R^{\left|K_{2}\right|}, \delta \in R, \eta_{1} \in R^{\left|J_{1}\right|}$, and $\eta_{2} \in R^{\left|J_{2}\right|}$ such that the following by Fritz John optimality conditions [18] are satisfied at $\left(\bar{x}^{1}, \bar{y}^{1}, \bar{x}^{2}, \bar{y}^{2}, \bar{z}^{1}, \bar{z}^{2}, \bar{p}, \bar{r}\right)$ 
Abstract and Applied Analysis

$$
\begin{aligned}
& \alpha^{T}\left(\nabla_{x^{1}} f\left(\bar{x}^{1}, \bar{y}^{1}\right)+\eta_{1}\right)+\nabla_{y^{1} x^{1}} f\left(\bar{x}^{1}, \bar{y}^{1}\right)\left[\beta-\alpha \bar{y}^{1}\right] \\
& +\nabla_{x^{1}}\left(\nabla_{y^{1} y^{1}} f\left(\bar{x}^{1}, \bar{y}^{1}\right) \bar{p}\right)\left[\beta-\alpha\left(\bar{y}^{1}+\frac{1}{2} \bar{p}\right)\right]=0, \\
& \alpha^{T}\left(\nabla_{x^{2}} g\left(\bar{x}^{2}, \bar{y}^{2}\right)+\eta_{2}\right)+\nabla_{y^{2} x^{2}} g\left(\bar{x}^{2}, \bar{y}^{2}\right)\left[\gamma-\delta \bar{y}^{2}\right] \\
& +\nabla_{x^{2}}\left(\nabla_{y^{2} y^{2}} g\left(\bar{x}^{2}, \bar{y}^{2}\right) \bar{r}\right)\left[\gamma-\delta \bar{y}^{2}-\frac{1}{2} \alpha \bar{r}\right]=0, \\
& \nabla_{y^{1} y^{1}} f\left(\bar{x}^{1}, \bar{y}^{1}\right)\left[\beta-\alpha\left(\bar{y}^{1}+\bar{p}\right)\right] \\
& +\nabla_{y^{1}}\left(\nabla_{y^{1} y^{1}} f\left(\bar{x}^{1}, \bar{y}^{1}\right) \bar{p}\right)\left[\beta-\alpha\left(\bar{y}^{1}+\frac{1}{2} \bar{p}\right)\right]=0, \\
& {\left[\nabla_{y^{2}} g\left(\bar{x}^{2}, \bar{y}^{2}\right)-\bar{z}^{2}\right][\alpha-\delta]+\nabla_{y^{2} y^{2}} g\left(\bar{x}^{2}, \bar{y}^{2}\right)\left[r-\delta\left(\bar{y}^{2}+\bar{r}\right)\right]} \\
& +\nabla_{y^{2}}\left(\nabla_{y^{2} y^{2}} g\left(\bar{x}^{2}, \bar{y}^{2}\right) \bar{r}\right)\left[\gamma-\delta \bar{y}^{2}-\frac{1}{2} \alpha \bar{r}\right]=0, \\
& \nabla_{y^{1} y^{1}} f\left(\bar{x}^{1}, \bar{y}^{1}\right)\left[\beta-\alpha\left(\bar{y}^{1}+\bar{p}\right)\right]=0, \\
& \nabla_{y^{2} y^{2}} g\left(\bar{x}^{2}, \bar{y}^{2}\right)\left[r-\delta \bar{y}^{2}-\alpha \bar{r}\right]=0, \\
& \beta^{T}\left[\nabla_{y^{1}} f\left(\bar{x}^{1}, \bar{y}^{1}\right)-\bar{z}^{1}+\nabla_{y^{1} y^{1}} f\left(\bar{x}^{1}, \bar{y}^{1}\right) \bar{p}\right]=0, \\
& r^{T}\left[\nabla_{y^{2}} g\left(\bar{x}^{2}, \bar{y}^{2}\right)-\bar{z}^{2}+\nabla_{y^{2} y^{2}} g\left(\bar{x}^{2}, \bar{y}^{2}\right) \bar{r}\right]=0, \\
& \delta\left(\bar{y}^{2}\right)^{T}\left[\nabla_{y^{2}} g\left(\bar{x}^{2}, \bar{y}^{2}\right)-\bar{z}^{2}+\nabla_{y^{2} y^{2}} g\left(\bar{x}^{2}, \bar{y}^{2}\right) \bar{r}\right]=0, \\
& \beta \in N_{D_{1}}\left(\bar{z}^{1}\right) \text {, } \\
& (\alpha-\delta) \bar{y}^{2}+\gamma \in N_{D_{2}}\left(\bar{z}^{2}\right), \\
& \eta_{1} \in C_{1}, \quad \eta_{1}^{T} \bar{x}^{1}=S\left(\bar{x}^{1} \mid C_{1}\right), \\
& \eta_{2} \in C_{2}, \quad \eta_{2}^{T} \bar{x}^{2}=S\left(\bar{x}^{2} \mid C_{2}\right), \\
& (\alpha, \beta, \gamma, \delta) \geq 0, \quad(\alpha, \beta, \gamma, \delta) \neq 0 .
\end{aligned}
$$

By hypothesis (i), (3.31) gives

$$
\beta=\alpha\left(\bar{y}^{1}+\bar{p}\right)
$$

Since $\nabla_{y^{2} y^{2}} g\left(\bar{x}^{2}, \bar{y}^{2}\right)$ is positive or negative definite, (3.32) yields

$$
\gamma=\delta \bar{y}^{2}+\alpha \bar{r}
$$


Suppose that $\alpha=0$, then (3.42) implies

$$
\gamma=\delta \bar{y}^{2}
$$

Using (3.42) in (3.30), we get

$$
(\alpha-\delta)\left[\nabla_{y^{2}} g\left(\bar{x}^{2}, \bar{y}^{2}\right)-\bar{z}^{2}+\nabla_{y^{2} y^{2}} g\left(\bar{x}^{2}, \bar{y}^{2}\right) \bar{r}\right]+\frac{1}{2} \nabla_{y^{2}}\left(\nabla_{y^{2} y^{2}} g\left(\bar{x}^{2}, \bar{y}^{2}\right) \bar{r}\right)\left[\gamma-\delta \bar{y}^{2}\right]=0,
$$

which on using hypothesis (iii) and $\gamma=\delta \bar{y}^{2}$ yields

$$
\alpha=\delta
$$

As $\alpha=0$, therefore the equations $\alpha=\delta$ and $\gamma=\delta \bar{y}^{2}$ give $\delta=0$ and $\gamma=0$, respectively. Further, (3.41) implies $\beta=0$. Consequently, $(\alpha, \beta, \gamma, \delta)=0$, contradicting (3.40). Hence, we have

$$
\alpha>0 .
$$

Subtracting (3.35) from (3.34) yields

$$
\left[r-\delta\left(\bar{y}^{2}\right)\right]^{T}\left[\nabla_{y^{2}} g\left(\bar{x}^{2}, \bar{y}^{2}\right)-\bar{z}^{2}+\nabla_{y^{2} y^{2}} g\left(\bar{x}^{2}, \bar{y}^{2}\right) \bar{r}\right]=0 .
$$

Using (3.42) and (3.46) in the above equation, we get

$$
\bar{r}^{T}\left(\nabla_{y^{2}} g\left(\bar{x}^{2}, \bar{y}^{2}\right)-\bar{z}^{2}\right)+\bar{r}^{T} \nabla_{y^{2} y^{2}} g\left(\bar{x}^{2}, \bar{y}^{2}\right) \bar{r}=0,
$$

which contradicts hypothesis (ii) unless

$$
\bar{r}=0 .
$$

Equation (3.42) yields

$$
\gamma=\delta \bar{y}^{2}
$$

Using (3.49) and (3.50) in (3.30), we obtain

$$
(\alpha-\delta)\left(\nabla_{y^{2}} g\left(\bar{x}^{2}, \bar{y}^{2}\right)-\bar{z}^{2}\right)=0,
$$

which on using hypothesis (iii) and (3.49) gives

$$
\alpha=\delta .
$$


Since $\alpha>0$, therefore

$$
\delta>0 .
$$

Now, using (3.41) and (3.46) in (3.29), we get

$$
\left(\nabla_{y^{1}}\left(\nabla_{y^{1} y^{1}} f\left(\bar{x}^{1}, \bar{y}^{1}\right) \bar{p}\right)\right) \bar{p}=0,
$$

which by hypothesis (iv) implies

$$
\bar{p}=0 .
$$

By (3.41) and (3.55), we have

$$
\beta=\alpha \bar{y}^{1}
$$

Using (3.46), (3.55), and (3.56) in (3.27), we get

$$
\nabla_{x^{1}} f\left(\bar{x}^{1}, \bar{y}^{1}\right)+\eta_{1}=0
$$

Equations (3.28), (3.46), (3.49), and (3.50) give

$$
\nabla_{x^{2}} g\left(\bar{x}^{2}, \bar{y}^{2}\right)+\eta_{2}=0
$$

and hence, we also have

$$
\left(\bar{x}^{2}\right)^{T}\left(\nabla_{x^{2}} g\left(\bar{x}^{2}, \bar{y}^{2}\right)+\eta_{2}\right)=0 .
$$

Thus, $\left(\bar{x}^{1}, \bar{y}^{1}, \bar{x}^{2}, \bar{y}^{2}, \bar{w}^{1}=\eta_{1}, \bar{w}^{2}=\eta_{2}, \bar{q}=0, \bar{s}=0\right)$ satisfies the dual constraints from (3.7) to (3.10), and so it is a feasible solution for the dual problem (SMND).

Further, using (3.46), (3.55), and (3.56) in (3.33), we obtain

$$
\left(\bar{y}^{1}\right)^{T} \nabla_{y^{1}} f\left(\bar{x}^{1}, \bar{y}^{1}\right)=\left(\bar{y}^{1}\right)^{T} \bar{z}^{1}
$$

Moreover, since $\beta=\alpha \bar{y}^{1}$ and $\alpha>0$, (3.36) implies $\bar{y}^{1} \in N_{D_{1}}\left(\bar{z}^{1}\right)$ so that

$$
\left(\bar{y}^{1}\right)^{T} \bar{z}^{1}=S\left(\bar{y}^{1} \mid D_{1}\right)
$$

From (3.37), (3.50), (3.52), and (3.53), we get

$$
\bar{y}^{2} \in N_{D_{2}}\left(\bar{z}^{2}\right) .
$$


Since $D_{2}$ is a compact convex set in $R^{\left|K_{2}\right|}$,

$$
\left(\bar{y}^{2}\right)^{T} \bar{z}^{2}=S\left(\bar{y}^{2} \mid D_{2}\right)
$$

Therefore, using (3.38), (3.39), (3.49), (3.55), (3.57), and (3.60)-(3.63), we obtain

$$
\begin{aligned}
f\left(\bar{x}^{1}, \bar{y}^{1}\right)+S\left(\bar{x}^{1} \mid C_{1}\right)+g\left(\bar{x}^{2}, \bar{y}^{2}\right)+S\left(\bar{x}^{2} \mid C_{2}\right)-\left(\bar{y}^{2}\right)^{T} \bar{z}^{2} \\
-\left(\bar{y}^{1}\right)^{T}\left[\nabla_{y^{1}} f\left(\bar{x}^{1}, \bar{y}^{1}\right)+\nabla_{y^{1} y^{1}} f\left(\bar{x}^{1}, \bar{y}^{1}\right) \bar{p}\right]-\frac{1}{2} \bar{p}^{T} \nabla_{y^{1} y^{1}} f\left(\bar{x}^{1}, \bar{y}^{1}\right) \bar{p} \\
-\frac{1}{2} \bar{r}^{T} \nabla_{y^{2} y^{2}} g\left(\bar{x}^{2}, \bar{y}^{2}\right) \bar{r} \\
=f\left(\bar{x}^{1}, \bar{y}^{1}\right)-S\left(\bar{y}^{1} \mid D_{1}\right)+g\left(\bar{x}^{2}, \bar{y}^{2}\right)-S\left(\bar{y}^{2} \mid D_{2}\right)+\left(\bar{x}^{2}\right)^{T} \bar{w}^{2} \\
\quad-\left(\bar{x}^{1}\right)^{T}\left[\nabla_{x^{1}} f\left(\bar{x}^{1}, \bar{y}^{1}\right)+\nabla_{x^{1} x^{1}} f\left(\bar{x}^{1}, \bar{y}^{1}\right) \bar{q}\right]-\frac{1}{2} \bar{q}^{T} \nabla_{x^{1} x^{1}} f\left(\bar{x}^{1}, \bar{y}^{1}\right) \bar{q} \\
\quad-\frac{1}{2} \bar{s}^{T} \nabla_{x^{2} x^{2}} g\left(\bar{x}^{2}, \bar{y}^{2}\right) \bar{s},
\end{aligned}
$$

that is, the two objective function values are equal.

Finally, from Theorem 3.1, we get that $\left(\bar{x}^{1}, \bar{y}^{1}, \bar{x}^{2}, \bar{y}^{2}, \bar{z}^{1}, \bar{z}^{2}, \bar{p}, \bar{r}\right)$ and $\left(\bar{x}^{1}, \bar{y}^{1}, \bar{x}^{2}\right.$, $\left.\bar{y}^{2}, \bar{w}^{1}, \bar{w}^{2}, \bar{q}, \bar{s}\right)$ are global optimal solutions for (SMNP) and (SMND), respectively.

Theorem 3.3 (Converse duality). Let $f: R^{\left|J_{1}\right|} \times R^{\left|K_{1}\right|} \rightarrow R$ and $g: R^{\left|J_{2}\right|} \times R^{\left|K_{2}\right|} \rightarrow R$ be differentiable functions, and let $\left(\bar{u}^{1}, \bar{v}^{1}, \bar{u}^{2}, \bar{v}^{2}, \bar{w}^{1}, \bar{w}^{2}, \bar{q}, \bar{s}\right)$ be a local optimal solution of (SMND). Suppose that

(i) the matrix $\nabla_{x^{1} x^{1}} f\left(\bar{u}^{1}, \bar{v}^{1}\right)$ is non singular,

(ii) $\nabla_{x^{2} x^{2}} g\left(\bar{u}^{2}, \bar{v}^{2}\right)$ is positive definite and $\bar{s}^{T}\left(\nabla_{x^{2}} g\left(\bar{u}^{2}, \bar{v}^{2}\right)+\bar{w}^{2}\right) \geq 0$ or $\nabla_{x^{2} x^{2}} g\left(\bar{u}^{2}, \bar{v}^{2}\right)$ is negative definite and $\bar{s}^{T}\left(\nabla_{x^{2}} g\left(\bar{u}^{2}, \bar{v}^{2}\right)+\bar{w}^{2}\right) \leq 0$,

(iii) $\nabla_{x^{2}} g\left(\bar{u}^{2}, \bar{v}^{2}\right)+\bar{w}^{2}+\nabla_{x^{2} x^{2}} g\left(\bar{u}^{2}, \bar{v}^{2}\right) \bar{s} \neq 0$,

(iv) one of the matrices $\left(\partial / \partial x_{i}^{1}\right)\left(\nabla_{x^{1} x^{1}} f\left(\bar{u}^{1}, \bar{v}^{1}\right)\right), i=1,2, \ldots,\left|J_{1}\right|$, is positive or negative definite.

Then, there exist $\bar{z}^{1} \in D_{1}$ and $\bar{z}^{2} \in D_{2}$ such that $\left(\bar{u}^{1}, \bar{v}^{1}, \bar{u}^{2}, \bar{v}^{2}, \bar{z}^{1}, \bar{z}^{2}, \bar{p}=0, \bar{r}=0\right)$ is feasible for (SMNP) and the objective function values of (SMNP), and (SMND) are equal. Furthermore, if the assumptions of weak duality (Theorem 3.1) are satisfied for all feasible solutions of (SMNP) and (SMND), then $\left(\bar{u}^{1}, \bar{v}^{1}, \bar{u}^{2}, \bar{v}^{2}, \bar{w}^{1}, \bar{w}^{2}, \bar{q}, \bar{s}\right)$ and $\left(\bar{u}^{1}, \bar{v}^{1}, \bar{u}^{2}, \bar{v}^{2}, \bar{z}^{1}, \bar{z}^{2}, \bar{p}, \bar{r}\right)$ are global optimal solutions for (SMND) and (SMNP), respectively.

Proof. It follows on the lines of Theorem 3.2. 


\section{Special Cases}

In this section, we consider some of the special cases of the problems studied in Section 3.

(i) If $J_{2}=\emptyset$ and $K_{2}=\emptyset$, then our problems (SMNP) and (SMND) reduce to the programs (PP) and (DP) studied in Gulati and Gupta [17].

(ii) If $J_{2}=\emptyset, K_{2}=\emptyset, C_{1}=\{0\}$ and $D_{1}=\{0\}$, then (SMNP) and (SMND) are reduced to the programs (SP) and (SD) studied in Gulati et al. [16] with the omission of nonnegativity constraints from (SP) and (SD).

(iii) If $J_{2}=\emptyset, K_{2}=\emptyset, p=0$, and $q=0$, then (SMNP) and (SMND) become a pair of symmetric nondifferentiable dual programs considered in Mond and Schechter [6] with the omission of nonnegativity constraints from the programs (P) and (D) studied in Mond and Schechter.

(iv) If $J_{2}=\emptyset, K_{2}=\emptyset, p=0, q=0, C_{1}=\{0\}$, and $D_{1}=\{0\}$, then the programs (WP) and (WD) of [15] are obtained with the omission of nonnegativity constraints from (WP) and (WD).

(v) If $J_{1}=\emptyset$ and $K_{1}=\emptyset$ in (SMNP) and (SMND), then the programs studied in [10] are obtained.

(vi) If $J_{1}=\emptyset, K_{1}=\emptyset, C_{2}=\{0\}$, and $D_{2}=\{0\}$ in (SMNP) and (SMND), then the programs (SP1) and (SD1) of [16] are obtained with the omission of nonnegativity constraints from (SP1) and (SD1).

(vii) If $J_{1}=\emptyset, K_{1}=\emptyset, r=0$, and $s=0$, then (SMNP) and (SMND) become a pair of symmetric nondifferentiable dual programs considered in [6] with the omission of nonnegativity constraints from the programs (P1) and (D1) studied in Mond and Schechter.

(viii) If $J_{1}=\emptyset, K_{1}=\emptyset, r=0, s=0, C_{2}=\{0\}$, and $D_{2}=\{0\}$, then (SMNP) and (SMND) become a pair of single objective symmetric differentiable dual programs considered in [15] with the omission of nonnegativity constraints from (MP) and (MD).

(ix) By eliminating the second-order and nondifferentiable terms, our problems (SMNP) and (SMND) reduce to the mixed symmetric dual programs studied by Chandra et al. [11] with the omission of $x^{1}>0, x^{2}>0, v^{1}>0$, and $v^{2}>0$ from the programs studied in Chandra et al. [11].

(x) By eliminating the second-order terms, our problems are reduced to the programs (MP) and (MD) studied in [12] with the omission of nonnegativity constraints from (MP) and (MD).

\section{Concluding Remarks}

It is to be noted that previously known results [6, 10-12,15-17] are special cases of our study. It is not clear whether the second-order mixed symmetric duality in mathematical programming can be further extended to higher-order multiobjective symmetric dual programs formulated in [19]. 


\section{Acknowledgments}

Ravi P. Agarwal and Izhar Ahmad are thankful to the King Fahd University of Petroleum and Minerals, Saudi Arabia for providing financial support to carry out this research.

\section{References}

[1] G. B. Dantzig, E. Eisenberg, and R. W. Cottle, "Symmetric dual nonlinear programs," Pacific Journal of Mathematics, vol. 15, pp. 809-812, 1965.

[2] B. Mond, "A symmetric dual theorem for non-linear programs," Quarterly of Applied Mathematics, vol. 23, pp. 265-269, 1965.

[3] M.S. Bazaraa and J. J. Goode, "On symmetric duality in nonlinear programming," Operations Research, vol. 21, pp. 1-9, 1973.

[4] S. Chandra and I. Husain, "Symmetric dual nondifferentiable programs," Bulletin of the Australian Mathematical Society, vol. 24, no. 2, pp. 295-307, 1981.

[5] S. Chandra, B. D. Craven, and B. Mond, "Generalized concavity and duality with a square root term," Optimization, vol. 16, no. 5, pp. 653-662, 1985.

[6] B. Mond and M. Schechter, "Nondifferentiable symmetric duality," Bulletin of the Australian Mathematical Society, vol. 53, no. 2, pp. 177-188, 1996.

[7] P. Kumar and D. Bhatia, "A note on symmetric duality for multiobjective non-linear program," Opsearch, vol. 32, no. 3, pp. 172-183, 1995.

[8] O. L. Mangasarian, "Second- and higher-order duality in nonlinear programming," Journal of Mathematical Analysis and Applications, vol. 51, no. 3, pp. 607-620, 1975.

[9] C. R. Bector and S. Chandra, "Second order symmetric and self-dual programs," Opsearch, vol. 23, no. 2, pp. 89-95, 1986.

[10] S. H. Hou and X. M. Yang, "On second-order symmetric duality in nondifferentiable programming," Journal of Mathematical Analysis and Applications, vol. 255, no. 2, pp. 491-498, 2001.

[11] S. Chandra, I. Husain, and Abha, "On mixed symmetric duality in mathematical programming," Opsearch, vol. 36, no. 2, pp. 165-171, 1999.

[12] X. M. Yang, K. L. Teo, and X. Q. Yang, "Mixed symmetric duality in nondifferentiable mathematical programming," Indian Journal of Pure and Applied Mathematics, vol. 34, no. 5, pp. 805-815, 2003.

[13] I. Ahmad, "Multiobjective mixed symmetric duality with invexity," New Zealand Journal of Mathematics, vol. 34, no. 1, pp. 1-9, 2005.

[14] C. R. Bector, S. Chandra, and Abha, "On mixed symmetric duality in multiobjective programming," Opsearch, vol. 36, no. 4, pp. 399-407, 1999.

[15] S. Chandra, A. Goyal, and I. Husain, "On symmetric duality in mathematical programming with F-convexity," Optimization, vol. 43, no. 1, pp. 1-18, 1998.

[16] T. R. Gulati, I. Ahmad, and I. Husain, "Second order symmetric duality with generalized convexity," Opsearch, vol. 38, no. 2, pp. 210-222, 2001.

[17] T. R. Gulati and S. K. Gupta, "Wolfe type second-order symmetric duality in nondifferentiable programming," Journal of Mathematical Analysis and Applications, vol. 310, no. 1, pp. 247-253, 2005.

[18] M. Schechter, "More on subgradient duality," Journal of Mathematical Analysis and Applications, vol. 71, no. 1, pp. 251-262, 1979.

[19] R. P. Agarwal, I. Ahmad, and A. Jayswal, "Higher order symmetric duality in nondifferentiable multiobjective programming problems involving generalized cone convex functions," Mathematical and Computer Modelling, vol. 52, no. 9-10, pp. 1644-1650, 2010. 


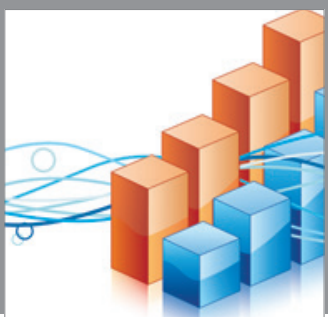

Advances in

Operations Research

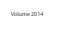

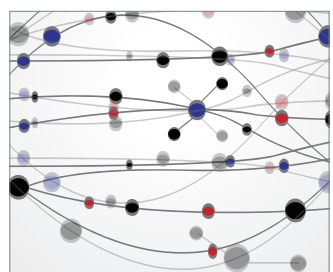

\section{The Scientific} World Journal
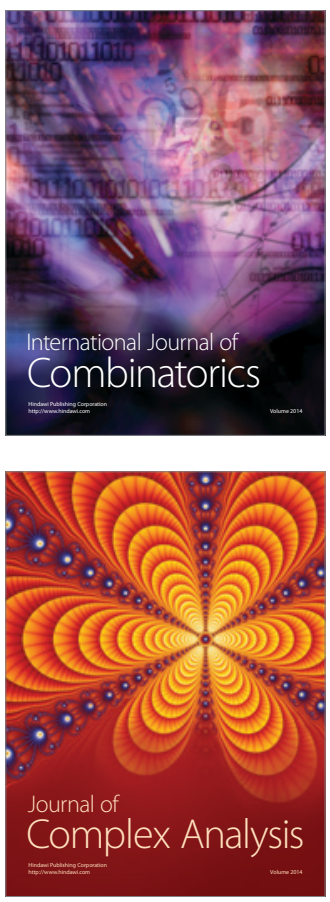

International Journal of

Mathematics and

Mathematical

Sciences
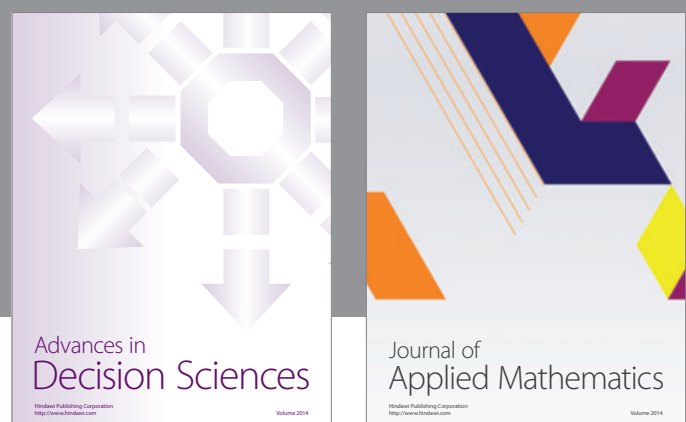

Journal of

Applied Mathematics
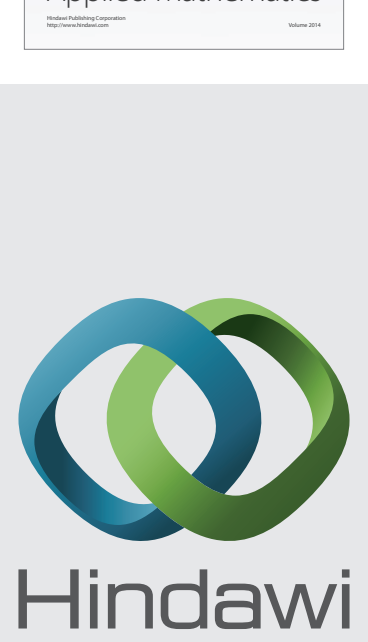

Submit your manuscripts at http://www.hindawi.com
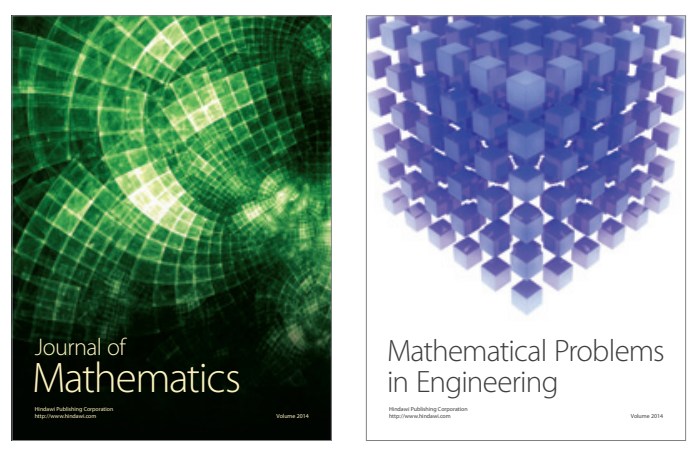

Mathematical Problems in Engineering
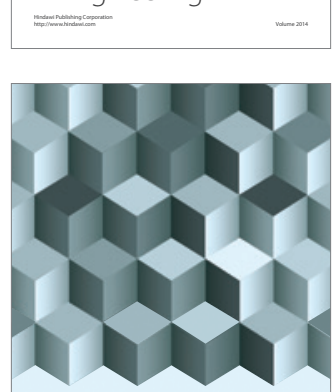

Journal of

Function Spaces
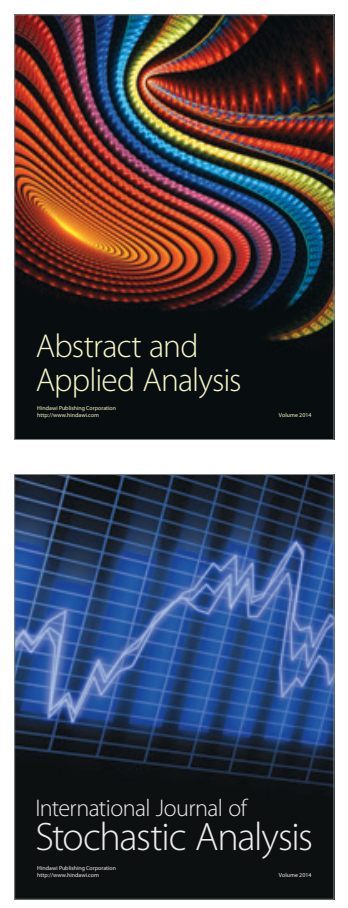

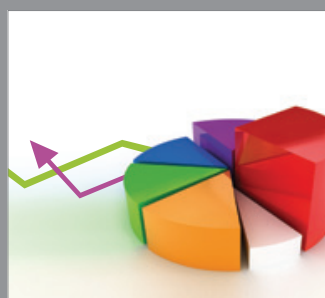

ournal of

Probability and Statistics

Promensencen
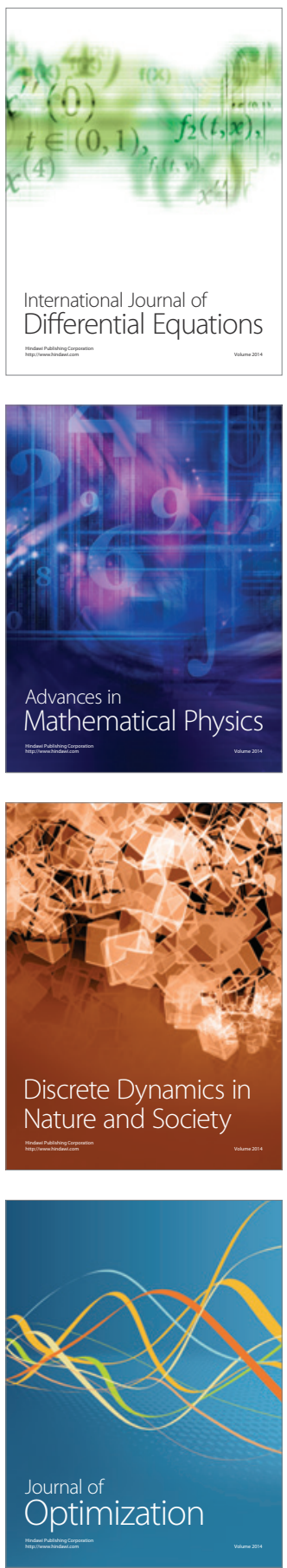